\title{
Model reduction based global optimization for large-scale steady state nonlinear systems
}

\author{
Min Tao ${ }^{1}$, Panagiotis Petsagkourakis ${ }^{2}, \mathrm{Jie} \mathrm{Li}^{1}$, and Constantinos Theodoropoulos ${ }^{1}$ \\ ${ }^{1}$ University of Manchester \\ ${ }^{2}$ University College London
}

July 7,2021

\begin{abstract}
Many engineering processes can be accurately modeled using partial differential equations (PDEs), but high dimensionality and non-convexity of the resulting systems pose limitations on their efficient optimization. In this work, a model reduction methodology combining principal component analysis (PCA) and artificial neural networks (ANNs) is employed to construct a reduced surrogate model, which is then utilized by advanced deterministic global optimization algorithms to compute global optimal solutions with theoretical guarantees. However, the optimization framework is still time-consuming due to the high nonconvexity of the activation functions inside the reduced ANN structure. To further enhance the capability of our optimization framework, two alternative strategies have been proposed. The first one is a piecewise-affine reformulation while the second one is based on deep rectifier neural networks with ReLU activation function. The performances of the two improved frameworks is demonstrated through two illustrative case studies.
\end{abstract}

\section{Hosted file}

PCA_ANN_GOP_to_AIChE_Journal.pdf available at https://authorea.com/users/424400/articles/ 529497-model-reduction-based-global-optimization-for-large-scale-steady-state-nonlinearsystems 